\title{
Failing to Ponder? Delusion-Prone Individuals Rush to Conclusions
}

\author{
Lars O. White and Warren Mansell* \\ Division of Psychology, School of Psychological Sciences, University of \\ Manchester, Manchester, UK
}

\begin{abstract}
Jumping to conclusions (JTC) has been proposed as an aetiological factor involved in the formation of delusions from the earliest stages. A number of researchers have thus shifted their focus to include the study of subclinical populations. Expanding on these studies, 17 delusion-prone and 22 control students completed four versions of the beads-in-a-jar paradigm (including multiple jar variants) to test recent claims regarding JTC's specificity to less ambiguous paradigms with a limited number of jars. Additional measures were administered to tease out a potential mechanism underlying JTC. The delusion-prone group showed a higher JTC bias which proved relatively robust across variants. Task performance was related to degree of self-reported rushing. It is concluded that delusion-prone individuals exhibit JTC, even when confronted with more ambiguous scenarios, potentially as a consequence of feeling rushed. Copyright (c) 2009 John Wiley \& Sons, Ltd.
\end{abstract}

\section{INTRODUCTION}

Garety, Bebbington, Fowler, Freeman and Kuipers (2007) postulate a critical role for the tendency of deluded individuals to hastily adopt beliefs despite insufficient data collection, comprising one part of the so-called jumping to conclusions (JTC) bias. This propensity may be analogous to the manner whereby delusional beliefs are developed and maintained on the basis of too little evidence.

Empirical data mainly derives from the beads task', a paradigm in which an experimenter draws beads from one of two containers filled with red and white beads in opposite ratios of $85: 15$, presenting them one at a time before replacing them. On the draws-to-decision (DTD) version of this task, participants view as many beads as they choose before deciding which jar was selected, whereupon the trial is terminated. A different procedure employs a fixed number of draws after each of which participants are requested to make probability estimates (PE) of bead origin for each

*Correspondence to: Dr Warren Mansell, School of Psychological Sciences, University of Manchester, Oxford Road, Manchester M13 9PL, UK.

Email: warren.mansell@manchester.ac.uk container, abolishing the terminating decision. On this variant, the number of draws to reach a decision or certainty functions as an index of jumping to conclusions (see Fine, Gardner, Craigie \& Gold, 2007).

The DTD procedure has relatively consistently demonstrated that $40-70 \%$ of delusional patients make decisions regarding jar-of-origin after only one or two draws (Dudley, John, Young \& Over 1997a; Freeman \& Garety, 2004; Peters \& Garety, 2006; Van Dael et al., 2006). In support, a recent meta-analysis has revealed DTD as the most reliable dependent measure of JTC (Fine et al., 2007). Moreover, the largest study to date, testing more than 100 delusional patients, found $53 \%$ and $41 \%$ to make a decision after just one or two draws on the standard 85:15 and a modified 60:40 version, respectively (Garety et al., 2005). On PE versions of the task, results have been less consistent, suggesting that delusions predispose to less data gathering despite probabilities being correctly estimated (see Fine et al., 2007).

The certainty estimates variant has also yielded the surprising result that delusional individuals revise their judgments more readily in response to disconfirmatory evidence (a bead of opposite colour) contradicting the popular notion of delusional incorrigibility (disconfirmatory bias; Garety, 
Hemsley \& Wessely, 1991; Peters \& Garety, 2006). It is noteworthy that Dudley et al. (1997a) have not supported this finding using a related paradigm.

Nevertheless, some researchers have incorporated a disconfirmatory bias (Freeman \& Garety, 2004; McKay, Langdon \& Coltheart, 2006) into JTC concept (Colbert \& Peters, 2002). Hence, JTC is defined as a proclivity towards premature decisionmaking as well as exaggerated revision of judgement following contrary evidence (Peters \& Garety, 2006). However, despite abundant research efforts, the underlying basis of JTC remains elusive.

\section{JTC as an Index of Deficient Sequential Processing or Impulsivity}

Initially, the emotionally neutral nature of the beads task was viewed as advantageous as it might isolate a reasoning deficit, independent of content or affect (Garety \& Hemsley, 1994). In this frame of mind, Garety et al. (1991) postulated that the JTC effect might be a product of lack of influence of stored regularities on current input (Hemsley, 1987, 2005). Increased fragmentation may lead to previously viewed evidence being less available (see Moritz \& Woodward, 2005). Indeed, especially in terms of the disconfirmatory bias, whereby past draws could be said to be neglected in favour of the most current one, this hypothesis appears particularly appealing (Garety et al., 1991).

Similarly, JTC is understandable in terms of individuals' impulsivity or motivation to rapidly complete the task. In discussing their findings of diminished focusing in deluded individuals (i.e., the ability to narrow down available hypotheses on the basis of integrating feedback across trials), Young and Bentall (1995) developed this idea further, suggesting a deficit in sequential processing. Thus, JTC may be adopted as a strategy by deluded patients to avoid premising their decisions on sequentially presented information. Despite, Fine et al.'s (2007) meta-analytic endorsement of this interpretation, this rationale cannot sufficiently account for findings showing that deluded individuals choose to see more evidence with increasing complexity of the beads ratios (Dudley et al., 1997a; Dudley, John, Young \& Over, 1997b; Menon, Pomarol-Clôtet, McKenna \& McCarthy, 2006; Young \& Bentall, 1997). Moreover, Moritz, Woodward and Lambert (2007) directly enquired the strategy individuals employed when completing the task, asking whether individuals were aiming to finish the task 'as soon as possible' and found no evidence for such a motivation.
Arguably, however, neither of these approaches adequately captures this potential mediating factor of JTC. Thus, Moritz et al.'s (2007) qualitative subjective assessment did not allow for graded responses regarding participants' inclination to finish the experiment soon. Moreover, as Dudley et al. (1997a) correctly note, their preclusion of impulsivity is predicated on a deduction from indirect evidence (i.e., impulsivity was not measured directly). Especially, in the light of Menon et al.'s (2006) finding that a substantial minority of deluded 'extreme responders' make a decision after one bead $(17 \%)$ irrespective of the difficulty level of the beads task employed (i.e., 60:40difficult/85:15-easy) and may thus 'carry' the JTC effect, an influence of motivation should not be ruled out prematurely.

\section{Is JTC Affect-Driven?}

In contrast to the rational (cold) perspective on the beads task offered by Garety and Hemsley (1994), Dudley et al. (1997b) have reintroduced emotion into the debate. Their attempt at elucidating JTC premises on studies employing meaningful and self-referent emotional versions of the beads task (Dudley et al., 1997b; Menon et al., 2006; Warman \& Martin, 2006; Warman, Lysaker, Martin, Davis \& Haudenschield, 2006; Young \& Bentall, 1997). Self-referent, emotional versions refer to alleged surveys about a person 'very much like' the subject, deriving from either a 'critical' or a 'friendly' group and requiring participants to guess which population sampled comments originate from (e.g., Warman \& Martin, 2006); Warman et al. (2006) recently adapted this paradigm further by asking individuals to imagine that the survey had actually been about themselves.

Dudley et al. (1997b) reported that the JTC bias was more pronounced (though not significantly) in the emotional version compared with a neutral and more realistic version which yielded similar means on DTD compared with their neutral beads findings (Dudley et al., 1997a). Similar findings in delusion-prone individuals, demonstrating selective significance for the self-referent version, have been reported by Warman and colleagues (Warman \& Martin, 2006; Warman et al., 2006; see below).

These findings indicate that delusional individuals may inappropriately generalize their behaviour on the self-referent and emotional version to the valence-neutral beads task. Possibly, this may occur as a result of incorrectly ascribing salience 
to neutral stimuli, thus discerning threat where others do not (Dudley et al., 1997b; Kapur, 2003). Notably, it is postulated that under threat, hasty decision-making generally becomes more adaptive (Dudley \& Over, 2003).

\section{Need for Closure and JTC}

Another intriguing account postulates that an elevated need for closure (NFC; Bentall \& Swarbrick, 2003) may motivate individuals to eschew uncertainty (Boyd \& Gumley, 2007) via JTC, especially, perhaps, when making inferential judgments where complete security is unobtainable (Dudley \& Over, 2003).

Evidence for this account, however, has been inconclusive at best. Thus, despite promising findings of significantly increased NFC in deluded and delusion-prone individuals, NFC itself was uncorrelated with performance on the beads task (Colbert \& Peters, 2002; Freeman et al., 2006; McKay et al., 2006). Moreover, even when employing a revised, more internally consistent measure of NFC, no significant difference between patients who displayed JTC (decision after one or two draws) and those who did not, was found on NFC (Freeman et al., 2006).

Interestingly, however, one recent study found a significant negative correlation $(\sim-0.3)$ between JTC with a similar construct, intolerance of uncertainty (IU; Freeston, Rhéaume, Letarte, Dugas \& Ladouceur, 1994), in a prodromal population using a more ambiguous $60: 40$ variant of the beads task (Broome et al., 2007). IU mainly dissociates from NFC with regard to measuring dysfunctional responses to uncertainty about the future, including, for instance, feeling paralyzed or inhibited by it (Buhr \& Dugas, 2002; Freeston et al., 1994). Unfortunately, another study, despite finding a significant difference on IU between paranoid patients and controls, found no evidence for a correlation with JTC. However, it should be noted that JTC itself showed no significant effect in this sample (Fraser, Morrison \& Wells, 2006).

More detrimental for this account, Freeman et al. (2006) report that elevated NFC in patients with non-affective psychosis is only indirectly associated with psychosis, via affect. Indeed, this suggests that NFC is non-specific to delusions as affect is conceived to impact on the full range of symptoms of psychosis. Crucially, this appears to rule out the specific and decisive contribution of NFC as 'motor' of delusion-formation (Freeman et al.,
2006). In a similar vein, it should also be noted that IU has been found increased in analogue obsessive-compulsive disorder (OCD; Holaway, Heimberg \& Coles, 2006). Although OCD patients can present with delusions (Beck \& Rector, 2005), they also display the reversal of JTC on the beads task, requesting more beads than controls before deciding (Fear \& Healy, 1997; but see also Moritz \& Woodward, 2005). This clearly begs the question whether a direct motivational account of JTC, in terms of IU or NFC, is feasible (Moritz \& Lincoln, unpublished document). Nevertheless, this account remains very popular (Colbert, Peters \& Garety, 2006), probably owing to its ability to reconcile seemingly contradictory findings of confusion and indecisiveness in (subclinical) delusions with a JTC bias (McKay et al., 2006; Moritz et al., 2007).

\section{JTC as an Artefact Resulting from a Liberal Acceptance (LA) Bias}

Although the preceding accounts diverge in various intricate ways, they share in common the interpretation of JTC as a reflection of hastiness in decision-making. By contrast, Moritz and Woodward (2004) have recently espoused the view that the bias results only under highly restricted conditions. Thereby, schizophrenic patients are putatively more receptive of hypotheses, thus exhibiting LA as opposed to homing in on one interpretation (JTC).

They suggest a decreased decision threshold (DDT; Moritz, Woodward \& Hausmann, 2006) resulting in premature, relatively undifferentiated acceptance of hypotheses. Hence, JTC merely emerges under artificial, unambiguous conditions of two mutually exclusive response-options as in the two-jar beads task, when evidence in support of one hypothesis simultaneously disconfirms its rival. Conversely, as Boyd and Gumley (2007) point out, decisions regarding the rejection or acceptance of delusional explanations are likely to be exceedingly difficult, involving discrimination between multiple contending hypotheses.

Consistent with this view, when instructed to appraise ambiguous picture stimuli, schizophrenic patients endorsed significantly more alternatives compared with controls, rather than preferring a single option as JTC seems to predict (Moritz \& Woodward, 2004). Similarly, when pictures were successively disambiguated, schizophrenic participants reduced their assigned probabilities to contending interpretations significantly less with 
respect to alternatives which were increasingly unlikely in the light of disconfirming evidence. As this pattern was coupled with a relatively intact incremented endorsement of the correct response, Moritz and Woodward (2006) postulated the presence of a specific bias against disconfirmatory evidence (BADE) in schizophrenia (Woodward, Moritz, Cuttler \& Whitman, 2006). Notably, a BADE has recently been observed in individuals suffering subthreshold schizotypy, consistent with its potential formative role (Buchy, Woodward \& Liotti, 2007).

In support of this interpretation, Moritz et al. (2007) demonstrated that deluded patients, exposing a JTC bias on a two-jar version, subsequently failed to show this bias on a four-jar version of the beads task. However, some methodological drawbacks as well as findings of a JTC effect on a three-jar version (Broome et al., 2007) plague this account (see Discussion).

\section{Delusion-Proneness and JTC}

Colbert and Peters (2002) and Warman and Martin (2006) accentuate the potential relevance of studying subclinical individuals high on delusional ideation (delusion-prone) as opposed to actual patients in order to investigate its involvement in delusionformation. Arguably, this condition, as generally indexed by the Peters et al. Delusion Inventory (PDI, Peters \& Garety, 1996), represents a premorbid state closely linked to delusions. Although Mullen (2003) has raised doubts regarding the delusionproneness construct, a number of points of overlap of delusion-proneness and clinical delusions (e.g., demographic variables, cognitive deficits), as well as links to schizotypy support its validity (Allen, Freeman, Johns \& McGuire, 2006; Peters, Joseph, Day \& Garety, 2004; Peters et al., 1999).

With regard to JTC, four studies have found evidence for a link between delusion-prone individuals and hasty decision-making, two of which have employed the beads task (Colbert \& Peters, 2002; Linney, Peters \& Ayton, 1998; McKay et al., 2006; Ziegler, Rief, Werner, Mehl \& Lincoln, 2008). However, Warman and Martin (2006), in the largest sample to date $(n=200)$, merely report an association between delusional ideation in the selfreferent versus neutral version when the first comment was negative $(r=-0.37)$. Similarly, Warman et al. (2006) merely found evidence for greater conviction in their decision regarding the jar-of-origin in their delusion-prone versus control group on the self-referent version. Third, Ziegler et al.'s (2008) finding of a correlation between delusion-proneness and hasty decisions on a conceptually similar letter recognition task failed to emerge on the beads task. In sum, these somewhat contradictory findings suggest that subthreshold delusional ideation may be linked to abnormal performance on the beads task or related paradigms, although critical determinants remain unclear.

\section{Specificity of JTC}

Commonly, JTC has been viewed as a specific contributor to delusion-maintenance and formation (Garety \& Freeman, 1999). Thus, evidence indicates that individuals with current or remitted delusions differ significantly on JTC from psychiatric controls (Dudley et al., 1997a; Garety \& Freeman, 1999). Moreover, Warman and Martin (2006) found a unique association between JTC and delusionproneness, as opposed to depressive and anxiety symptoms, corroborating specificity from an early stage onwards. However, Young and Bentall (1997) reported a marginally significant JTC (disconfirmatory) bias in both their deluded patients and their depressed controls compared with controls casting doubt on JTC's specificity and strengthening the case for further investigation.

In sum, the critical moot points are as follows:

1. Underlying construct

a. To what extent does JTC reflect impaired 'cold' executive reasoning processes as opposed to 'hot' affect-dependent processes?

b. Is JTC attributable to a defensive reaction against ambiguity and/or a need for closure?

c. Is JTC restricted to relatively artificial paradigms using two jars/hypotheses where the likelihood of both are inversely related?

2. Aetiological relevance

a. Does it precede delusions, i.e., present in subclinical delusions?

b. Does it specifically co-occur with, and may thus plausibly promote formation of delusional beliefs, as opposed to other theoretically unrelated symptoms?

\section{HYPOTHESES}

Primarily, the focus was on Moritz and Woodward's (2004) proposition of JTC as a methodological artefact of employing two jars and its consequent elimination in a multiple jar beads task. Thus, 
four versions of the beads task were employed, featuring two, three and four jars with varying ratios. However, due to drawbacks in Moritz et al.'s (2007) study (see below), and Broome et al.'s (2007) findings to the contrary, contrasting results were predicted.

1. It was hypothesized that delusion-prone participants would jump to conclusions irrespective of beads task variant.

2. A significant difference between groups on NFC and IU was also predicted which would correlate with JTC, echoing Broome et al.'s (2007) findings. Additionally indecisiveness was gauged and predicted to coincide with participants' decision-making style.

3. Third, relative specificity of the JTC bias to delusion-proneness as opposed to other subthreshold conditions was expected.

4. Finally, the individuals' haste in completing the experiment ('rushing') and its potential effect on JTC was investigated in an exploratory fashion.

\section{METHOD}

\section{Group Classification}

Consistent with previous research (Colbert \& Peters, 2002; Green et al., 2001; Laroi \& van der Linden, 2005; Linney, et al., 1998), individuals were categorized as either delusion-prone or control based on their high and low PDI-21 scores, respectively. Specifically, Colbert and Peters' (2002) cut-off $(>75.5)$ was utilized as an orientation point for the delusion-prone group. However, in order to render the control group more representative of the population, Linney et al.'s (1998) wider cut-off $(<61)$ was employed although the mean control PDI-21 score was still below, and the majority of controls (19) scored within Colbert and Peters' control cut-off $(<34.5)$, securing comparability.

\section{Design}

A non-randomized matched groups mixed design was employed, allowing comparison of the delusion-prone to a control group. For the first part, the independent variables were level of delusional ideation and beads task version. Number of marbles requested represented the dependent variable. Twenty-four individuals were assigned to the control and 20 to the experimental groups.

\section{Participants}

Three hundred sets of questionnaires, including the PDI-21 were distributed among an opportunity sample of university students, largely first year psychology students recruited via e-mail, posters and after lectures. The experimenter, initially remaining blind regarding participants' group status, invited respondents scoring within the cut-offs to participate in the experiment in return for course credit, if applicable.

Forty-four individuals (mean age $=19.73$; standard deviation $[S D]=3.30$; 37 females, 7 males) completed the full experiment. A potential effect of 'rushing' was noted after the fourth participant, whereupon it was included as an additional variable, resulting in 40 participants having a complete dataset. In order to preserve the comparability of the sample before and after controlling for the potential effect of 'rushing', these first four participants were excluded from the analyses.

The blind was lifted after 19 participants (12 control; 7 experimental) to facilitate recruitment and allow for randomization of beads task variants. ${ }^{1}$

\section{Materials and Apparatus}

The participant information sheet (PIS), consent form, the instructions for the beads task and the picture prediction task as well as the prediction response sheets were provided in paper format.

\section{Beads Task}

To ease understanding, the experiment commenced with the standard version, followed by the three variants in a randomized order conforming to identical procedures: bottles filled with coloured marbles of the respective ratios (see below) and labelled A-B/C/D were presented. After hiding the bottles behind the screen, the experimenter alleged to sample beads from one of them, one at a time. The sample actually adhered to a pseudorandom order to allow for standardization. After

\footnotetext{
${ }^{1}$ The experimenter administered beads task and the variable 'rushing' were considered potentially susceptible to a Rosenthal effect, as these could plausibly be influenced by participant-experimenter interaction. In the experimental group, five participants with complete datasets were tested before the blind was lifted, whereas this applied to 10 controls. Neither of these groups scored significantly differently to their counterparts in their respective groups on these variables $(p>0.3)$.
} 
presenting a marble to the participant, the experimenter pretended to replace it. Following each draw, the participant was required to signal if another marble was to be drawn or whether a decision had been reached by pointing to either of the two signs reading 'More items please' or 'No more items, I have decided'. A decision terminated the trial and both the decision and the number of beads requested were noted.

For all variants of Phillips and Edwards' (1966) beads task, clear wine bottles were utilized, filled with the following ratios of coloured marbles. The standard two-jar version used red and green marbles in opposite ratios of $85: 15$. In the modified two-jar version, the bottles contained white and green marbles in reverse ratios of $60: 40$. The threejar version utilized white, black and red marbles in the three possible permutations of the ratio $44: 28: 28$. In the final four-jar version, the bottles contained the following ratios of blue $(B)$, green (G) and yellow marbles (Y): 90G:10B; 50G:50B; 90B:10G; 90Y:10B. In order to obtain the predetermined sampling orders for the standard two-jar and four-jar versions, the respective papers were consulted (Huq, Garety \& Hensley, 1988; Moritz et al., 2007), and those for two remaining versions were retrieved from Matthew Broome. To allow the bottles to be hidden from view, a screen was set up between the participant and the experimenter. Two signs reading 'More items please' and 'No more items, I have decided' were attached to this screen as well as a transparent sachet containing the signs featuring the respective ratios of each beads task version, to reduce memory load. Decisions were recorded by the experimenter on a checklist.

\section{Symptom Measures}

Peters et al.'s Delusions Inventory, 21-Item Version (PDI-21; Peters et al., 2004)

The PDI was developed to measure delusionlike experiences in the normal population (delusional ideation), gauging the degree of 'delusion-proneness'. The items encompass questions such as 'Do you ever feel as if you were being persecuted in some way?' or 'Do you ever feel that you are a very special or unusual person?' (Yes/No). Following a 'Yes' response, individuals are requested to indicate the extent of distress, preoccupation and conviction associated with the respective item on five-point scales. Allotting one point for every 'Yes' and adding respective subscale ratings yield the total score ranging from 0 to
336. The PDI-21 possesses acceptable psychometric properties (Peters et al., 2004).

Depression Anxiety Stress Scales, 21-Item Version (DASS-21; Lovibond E Lovibond, 1995)

The DASS was expressly developed to gauge and differentiate between depression and anxiety in the normal population (Crawford \& Henry, 2003). It consists of three subscales comprising seven items each, measuring distress (S), anxiety (A) and depression (D), the first scale representing shared whereas the latter two scales constituting specific characteristics of anxious and depressive symptomatology, respectively. Scores for the full measure range from 0 to 63 and 0 to 21 for each subscale. The DASS-21 enquires to what degree statements, such as 'I tend to over-react to certain situations' (S), 'I found it difficult to work up the initiative to do things' (D) or 'I felt scared without any good reason' (A) applied to the respondent during the past week, providing a four-point Likert scale ranging from 0 (Did not apply to me at all) to 3 (Applied to me very much, or most of the time) for their answers. Antony, Bieling, Cox, Enns and Swinson (1998) report adequate psychometric qualities.

\section{Mood Disorder Questionnaire (MDQ; Hirschfeld et al., 2000)}

The MDQ screens for lifetime symptoms and behaviours associated with a manic or hypomanic syndrome via response to 13 yes/no items. The final item enquires the consequent level of functional impairment on a four-point scale ('no problem' to 'serious problem'). A sample item represents, 'Has there ever been a period of time when you were not your normal self and you felt much more self confident than usual?' Hirschfeld et al. (2000) reported good sensitivity and excellent specificity for the MDQ.

\section{Process Measures}

Need for Closure Scale (NFCS; Kruglanski, Webster \& Klem, 1993)

The NFCS aims to gauge respondents' motivated desire for an answer as opposed to ambiguity and uncertainty (Kruglanski \& Webster, 1996). Its 42 items comprise five subscales measuring preference for order and structure (e.g., 'I hate to change my plans last minute'), preference for predictability in future contexts (e.g., 'I don't like to be with people who are capable of unexpected actions'), decisive- 
ness (e.g., 'I usually make important decisions quickly and confidently'), discomfort with ambiguity (e.g., 'I dislike it when a person's statement could mean many different things') and closedmindedness (e.g., 'I feel irritated when one person disagrees with what everybody else in a group believes'). Responses are made on a six-point Likert scale ranging from 1 (disagree) to 6 (agree). Total scores are calculated by summing scores on each scale. Notably, however, recent findings (Colbert, Peters \& Garety, 2006; Freeman et al., 2006) have reported a negative correlation of the decisiveness subscale, as well as a lack of an association of the closed-mindedness subscale, with the other NFCS subscales. Hence, these researchers excluded these two scales resulting in the NFCS-R, whose results are also reported in this study.

\section{Intolerance of Uncertainty Scale (IUS; Freeston et al., 1994)}

The IUS comprises 27 statements relating to cognitive appraisal of and emotional and behavioural responses to uncertainty. Sample items represent 'When it's time to act uncertainty paralyses me' or 'Being uncertain means that a person is disorganised'. Participants are required to determine the extent to which the statement applies to them on a five-point scale ranging from 1 (Not at all characteristic of me) to 5 (Entirely characteristic of me). The English version demonstrates adequate psychometric characteristics (Buhr \& Dugas, 2002).

\section{Frost Indecisiveness Scale (FIS; Frost $\mathcal{E}$}

\section{Shows, 1993)}

The FIS comprises 15 items targeting difficulties with making decisions and indecisiveness, allowing for responses on a Likert scale ranging from 1 (strongly disagree) to 5 (strongly agree). The FIS consists of two subscales gauging Fears about Decision-Making and Positive Decision-Making, exemplified by questions such as 'I often worry about making the wrong choice' or 'I always know exactly what I want', respectively. A total score is derived by reversing the scores of the latter subscale and adding them to those of the former. Frost and Shows (1993) report adequate psychometric properties for the FIS.

\section{Rushing}

Participants were asked to indicate the extent to which they were rushing on a visual analogue scale, ranging from 0 (not at all rushed) to 100 (extremely rushed). Specifically, the item enquired 'How much in a rush were you to finish this study' so as to control for a potential confounding effect of time-pressure on either of the experimental tasks.

\section{Procedure}

Initially, participants received the PIS, consent forms and the beads task instructions. To ease understanding, the experiment commenced with the standard version, followed by the three variants in a randomized order conforming to identical procedures. Individuals were then handed new instructions before completing the 'Zoom!' task, the results of which will be reported in a separate paper. Next, participants were administered the process, symptom (including a second 'control assessment' of PDI delusion-proneness) and rushing measures, respectively. After this, they were informally asked for the grounds of their decision on the bottles task. Finally, if applicable, credit sheets were signed and participants were debriefed.

\section{Analyses}

In contrast to some previous studies (e.g., Huq et al., 1988), more conservative two-tailed tests were employed throughout. NFCS, IUS, FIS, Rushing, DASS and MDQ scores were entered as covariates in separate analyses, provided they yielded a difference at least at non-significant trend level between the groups.

\section{RESULTS}

\section{Population}

The sampling population $(n=223)$ comprised 37 males and 186 females with a mean age of 19.73 $(\mathrm{SD}=4.84)$. Respondents scored, on average, 49.24 $(\mathrm{SD}=35.84)$ on the PDI-21 and within a range of $0-73$. Thus, both the average PDI-21 score and the SD were lower compared with the general population norms established by Peters et al. (2004; $\mathrm{M}=58.9 ; \mathrm{SD}=48.0)$. PDI scores did not differ as a function of gender $\mathrm{t}(221)=0.399, p>0.05$, and were not significantly correlated with age $(r=$ $-0.12, p>0.05)$. However, a low significant positive correlation did emerge between DASS and PDI $(r=0.215, p=0.001)$.

\section{Sample}

The sample itself consisted of 44 individuals, four of which were excluded due to incomplete 
Table 1. Means and standard deviations (SD) on measures for the control and delusion-prone group

\begin{tabular}{lcrc}
\hline & Controls Mean (SD) & Delusion-prone Mean (SD) & Significance \\
\hline Age & $19.50(2.30)$ & $19.35(1.66)$ & $p>0.1$ \\
FIS total & $46.82(20.85)$ & $42.06(13.96)$ & $p>0.1$ \\
NFCS total & $149.27(23.55)$ & $147.95(28.41)$ & $p>0.1$ \\
NFCS-R & $102.41(24.60)$ & $103.84(24.27)$ & $p>0.1$ \\
IUS & $54.73(18.80)$ & $73.88(24.72)$ & $p=0.009^{* *}$ \\
Rushing & $25.05(15.98)$ & $39.18(24.17)$ & $p=0.035^{*}$ \\
DASS & $9.09(6.87)$ & $19.00(12.33)$ & $p=0.003^{* *}$ \\
MDQ & $6.32(3.578)$ & $8.44(3.405)$ & $p=0.087^{* *}$ \\
PDI-21 & $26.41(10.28)$ & $109.96(32.34)$ & $p<0.001^{* *}$ \\
PDI-21 1 Exp & $22.77(17.70)$ & $71.82(33.16)$ & $p<0.001^{* *}$ \\
\hline
\end{tabular}

$* p<0.05 .{ }^{* *} p<0.01 .{ }^{* * *} p<0.1$.

FIS $=$ Frost Indecisiveness Scale. NFCS $=$ Need for Closure Scale. IUS $=$ Intolerance of Uncertainty Scale. DASS $=$ Depression Anxiety Stress Scales. MDQ = Mood Disorder Questionnaire. PDI = Peters et al. Delusion Inventory.

datasets (see Method). Additionally, one participant represented an outlier, lying above $3.29 \mathrm{SD}$ above the mean on age and the 60:40 version of the beads task and was consequently omitted. ${ }^{2}$ This was further justified by Warman and Martin's (2006) finding of age as an extraneous variable on the beads task. Thus, a total of 39 participants (17 experimental, 22 control; 6 male, 33 female) were submitted to further analyses. Low and high scorers did not differ significantly on age, $F(1,37)=$ $0.049 ; p=0.825)$. However, the experimental group contained significantly more males than the control group $\left(\chi^{2}=4.27, \mathrm{df}=1, p<0.05\right){ }^{3}$

So as to assess group differences on questionnaire measures (see Table 1), a multivariate analysis of variance was conducted. In line with the predictions, the IUS yielded a highly significant difference $(F[1,37]=7.571 ; p<0.01)$, with delusion-prone individuals, on average, scoring significantly higher than the controls. ${ }^{4}$ Delusion-prone individuals were also, on average, significantly more rushed than controls, $F(1,37)=4.493 ; p<0.05$. Neither the FIS $(F[1,37]=0.657, p>0.05)$, nor NFCS-R $(F[1,37]$ $=0.033, p>0.05)$ nor the NFCS $(F[1,37]=0.087, p>$ $0.05)$ reached significance $(p>0.05)$.

With respect to symptom measures, the mean DASS-21 score of the experimental group exceeded that of con-

\footnotetext{
${ }^{2}$ A sensitivity analysis was conducted, by calculating results with and without the outlier (see below).

${ }^{3} \mathrm{~A}$ series of one-way analyses of variance were conducted to test for potential effects of gender on those dependent measures which yielded significant effects (Beads task, Confusion, DASS, IUS, Rushing). None of these were found significant $(p<0.05)$ nor at trend level $(p<0.1)$.

${ }^{4}$ However, in order to test for specificity, DASS-21 scores were entered as a covariate, which reduced this difference well below trend-level, $F(1,36)=0.104, p=0.749$.
}

trols, a difference which was found highly significant, $\mathrm{F}(1,36)=10.773, \mathrm{p}=0.003$. The $M D Q$ yielded a nonsignificant trend, $\mathrm{F}(1,37)=3.090, \mathrm{p}=0.087$, with the mean experimental group score being elevated above that of controls. In confirmation of participants' group status, mean experimental scores on the day were significantly higher than those of controls on the PDI, $\mathrm{F}(1,37)=21.602, \mathrm{p}<0.001$.

\section{Beads Task}

Analysis 1. Group Differences on JTC

The mean number of beads requested by the experimental group was lower than for controls on all variants of the beads task. A $2 \times 4$ mixed analysis of variance (ANOVA) was conducted with group as a between and beads task version as the within subjects independent variables and number of requested beads as the dependent variable. Mauchly's Test indicated that sphericity could not be assumed (Mauchly's W $=0.463$, df $=5, p<0.001)$, resulting in the use of corrected Greenhouse-Geisser tests. A significant effect of beads task variant on number of draws was found, $F(1.980,73.267)=48.488, p<0.001$, indicating that individuals modified the amount of beads viewed in response to the task variant. Sidak post hoc tests showed that the number of beads viewed by participants differed significantly among all versions $(p<0.05)$. In particular, participants chose to view most evidence on the three-jar, followed by the $60: 40$, the four-jar and the $85: 15$ versions, respectively.

Consistent with the predictions, a significant effect of group on number of requested beads was found $(F[1,37]=5.475, p=0.025)$, with Sidak post 


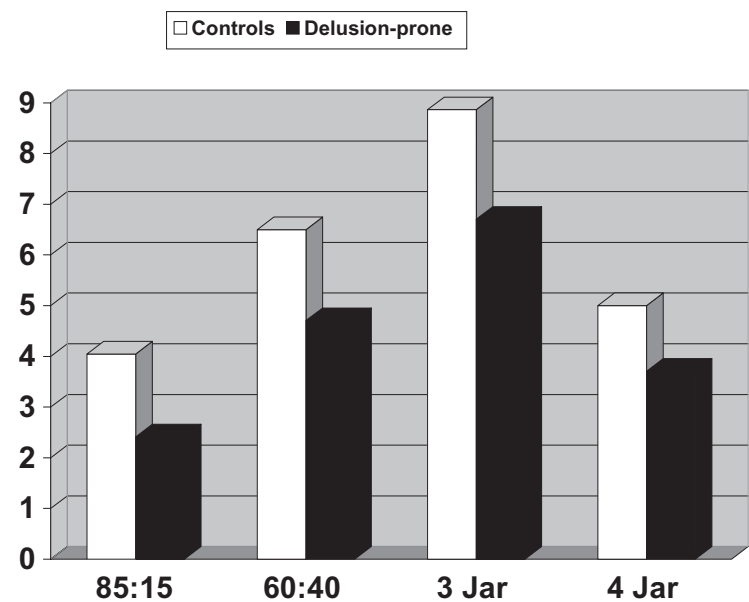

Figure 1. Estimated marginal means for number of draws by task version for both groups

hoc analyses indicating that controls, on average, requested significantly more beads $(M=6.10$, standard error $[\mathrm{SE}]=0.49)$ than experimental subjects $(\mathrm{M}=4.38, \mathrm{SE}=0.55)$. No significant interaction between group and version was observed ( $F[1.980$, 73.276] $=0.409, p=0.664$ ), implying that performance did not differ as a function of task variant between the groups (see Figure 1). Consequently, scores were collapsed across variants to create a composite index of JTC, ${ }^{5}$ yielding a large effect for the difference between groups $(\mathrm{d}=0.817)$.

\section{Analysis 2. Correlates of JTC}

In a further step, correlational analyses were conducted to explore the links between the beads task and the standardized measures. No significant correlations were found with any of these measures. However, the self-report measure 'Rushing' correlated significantly with the JTC composite index, $r(39)=0.349, p=0.029$. Hence, rushing was considered as a covariate in order to explore the mechanism underlying JTC.

\section{Analysis 3. Effect of 'Rushing' on JTC}

In an exploratory analysis, 'Rushing' was entered as a covariate into the $2 \times 4$ mixed ANOVA performed previously, testing the effect of group by

\footnotetext{
${ }^{5}$ Follow-up analyses yielded high correlations $(>0.7)$ between all beads tasks and medium to large effects on all versions which were also significant with the exception of the threejar variant.
}

beads task variant on number of requested beads. This resulted in a reduction of the effect of group below significance, $F(1,36)=3.062, p=0.104)$.

\section{Analysis 4. Sensitivity Analysis}

Repeating the analyses with the outlier included led to a reduction of the composite JTC, $F(1,38)=$ $3.692, p=0.062$, and its correlation with rushing, $r(40)=-0.282, p=0.078$, to a trend level.

\section{DISCUSSION}

In accordance with the predictions, delusion-prone individuals displayed a large JTC effect, robust to variation in jar or hypothesis number. Moreover, the effect emerged despite a more conservative two-tailed comparison with a control group that more closely resembled the general population. As such, Colbert and Peters' (2002) findings were both replicated and shown to extend to more complex and multiple jar versions of the beads task.

\section{LA and JTC}

With regard to the underlying basis of JTC, these data conflict with Moritz et al.'s (2007) liberal acceptance account of beads task findings, whereby a decreased threshold for acceptance only results in JTC on the mutually exclusive two-jar version. Notably, however, Moritz et al. (2007) predicated their conclusion on the absence of an effect (on the four-jar versus the two-jar version) without finding evidence for a task by variant interaction, clearly weakening their argument. Importantly, the absence of this interaction was replicated.

Furthermore, this was supplemented by a large effect on a composite index of all task versions, which also individually correlated highly with one another and, without exception, yielded medium to large effect sizes. Evidently, this contrasts with Moritz et al.'s (2007) data and conflicts with a qualitative difference between scenarios featuring two versus multiple hypotheses. Methodologically, their four-jar version diverged from the standard procedure (and their own two-jar versions) in that a 'decision' was equated with 'absolute certainty' on a five-point certainty scale and did not terminate the trial. In doing so, their set-up arguably resembled the less reliable certainty estimates procedure more closely than DTD (see Garety \& Freeman, 1999). Since research employing a fixed number of draws has yielded a JTC effect less consistently (Fine et al., 2007; Garety \& Freeman, 
1999), it has been argued that the terminating decision constitutes a critical variable within the beads task procedure, increasing the incentive to strike decisions (Dudley et al., 1997a).

This latter conclusion is of particular interest as it may help to explain the apparent discrepancies between the research of Moritz, Woodward and colleagues (e.g., Moritz \& Woodward, 2004) and that by Garety and co-workers (e.g., Garety et al., 1991). As such, although a liberal acceptance account may correctly suggest that delusional individuals accept multiple hypotheses simultaneously due to a lowered acceptance criterion, Moritz et al.'s research has generally not involved a decision stage. With regard to their picture disambiguation paradigm, for instance, all that was required of participants was to rate the plausibility of each interpretation (Moritz et al., 2006). On a theoretical level, it is possible that they were thus tapping an earlier stage of belief formation, i.e., assessment of each hypothesis by itself without a summary appraisal or a final choice (see Fischhoff \& BeythMarom, 1983).

Furthermore, their research may also be explicable in the light of Dudley and Over's (2003) postulation that the JTC effect may result from the inappropriate attribution of threat to neutral material. As Fine et al. (2007) noted, Dudley and Over's (2003) hypothesis of a threat confirmation strategy in delusional patients, would result in premature decision-making (i.e., JTC) when possible, but not necessarily in greater certainty regarding hypotheses. To clarify, exaggerated threat perception may eventuate in premature endorsement of a hypothesis 'just to be on the safe side', despite a comparable level of certainty to someone with normal threat perception. As such, these considerations may account for their failure to find evidence for JTC in their research which generally employs plausibility judgments (e.g., Buchy et al., 2007).

\section{NFC, IU and JTC}

The present findings of elevated IU in delusionprone individuals, but a simultaneous lack of a significant difference in NFC, are initially somewhat surprising and contradictory, as both constructs have been viewed along similar lines (e.g., Broome et al., 2007; Moritz \& Lincoln, unpublished manuscript). In the following, these results will first be considered separately. Notably, the negative NFC finding contrasts with a number of previous studies. Thus, various researchers have found elevated levels of NFC or one of its subscales in delusion-prone and clinical populations (Bentall \& Swarbrick, 2003; Colbert \& Peters, 2002; Colbert, Peters \& Garety, 2006; Freeman et al., 2006; McKay et al., 2006). Thus, the present negative findings may have been due to a lack of power.

In contrast, the positive finding of increased IU in delusion-proneness is congruent with previous findings, reporting elevated IU in prodromal and paranoid individuals (Broome et al., 2007; Fraser, et al., 2006). However, these two studies diverged in that one found a significant association between IU and DTD (Broome et al., 2007), whereas the other did not (Fraser et al., 2006). In this respect, the current data are more consistent with the latter, as no correlation between DTD and IU was observed, contradicting the notion of IU as a determinant of JTC. Furthermore, insofar as NFC and IU may reflect similar constructs, this finding also accords with the unanimously reported lack of a relationship between NFC and DTD (Colbert \& Peters, 2002; Freeman et al., 2006; McKay et al., 2006).

This interpretation gains further impetus with respect to the elimination of the strong group difference in IU when partialling out general psychopathology. Interestingly, a similar finding was documented by Freeman et al. (2006) for NFC. In other words, this implies that both IU and NFC may represent non-specific transdiagnostic processes (see Harvey, Watkins, Mansell \& Shafran, 2004). As such, the most parsimonious interpretation is that IU and NFC are not crucial determinants necessary for the development of specific symptoms such as delusions. This conclusion draws from the consideration that if indeed a transdiagnostic IU or NFC were partially responsible for JTC, then JTC should be distributed across symptom presentations, rather than exclusively co-occurring with a single symptom (see Harvey et al., 2004). In turn, these considerations provide support for the specificity of JTC to delusions, consistent with its relevance as a mechanism of delusion-formation.

To summarize, these data do not support a role either for NFC or IU in directly governing JTC. Tentatively, they also support a differential treatment of NFC and IU, with the latter, perhaps, reflecting a more sensitive measure to variations in delusional ideation and general psychopathology.

\section{Indecisiveness and JTC}

Contrary to the predictions, high indecisiveness failed to associate with increased DTD on the beads 
task. Although this result is somewhat surprising, as the beads task expressly taps decision-making (Garety \& Hemsley, 1994), the discrepancy may mirror the divergence of decision-making in, and outside, the laboratory, as the FIS attempts to gauge real-life, meaningful decisions, which bear consequences for the individual (Frost \& Shows, 1993).

It should also be noted that the finding of a lack of difference in indecisiveness between delusionprone and control participants is at odds with McKay et al.'s (2006) findings which may, however, be due to compromised power. Surprisingly, the latter research group actually reported a positive association between indecisiveness on a different measure and delusional ideation, interpreting this as an instance of greater vacillation and an indicator of jumping to new conclusions.

\section{Specificity of JTC}

Congruent with the predictions, JTC emerged as relatively specific to delusion-proneness, as indexed by the lack of significant correlations with general psychopathology and hypomanic symptoms. This finding concords with Warman and Martin's (2006) study, documenting no association between JTC and depression or anxiety.

It also confirms delusion-proneness as a construct separate from general psychopathology, depression or anxiety which is, at least, associated with its own reasoning profile. As such, it provides some preliminary grounds to counter the scepticism expressed by some researchers regarding the degree of overlap between delusion-proneness and clinical delusions (c.f. Mullen, 2003; Preti et al., 2007).

\section{'Rushing' and JTC}

These preliminary findings tentatively suggest that the extent to which an individual is rushing predicts his/her level of JTC. Initially, this may give rise to doubts regarding the validity of previous findings, which may simply be an artefact of being in a hurry to finish the task. However, given that experimental subjects also rushed more than controls, the data provide provisional support for its involvement in facilitating JTC.

The finding markedly contrasts with Moritz et al.'s (2007) data. It is noteworthy, however, that no graded responses were possible in their assessment of this factor. Conversely, a plausible explanation of the current data is that partly rushing may accelerate decision-making on the beads task.

Interestingly, a study from within basic cognitive reasoning literature has found similar results for non-clinical individuals. Thus, Heit (1998) observed that time-pressure led individuals to weight information contradicting their prior expectancies less, indicating that it led to consideration of less data. Intriguingly, a recent control model of errors in physicians' writing of prescriptions suggested that limited time appears to have the single most deleterious effect on the occurrence of errors (Marken, 2003). In this context, it is noteworthy that although 'cognitive errors' or false beliefs (delusions) are assumed to result from hastier decision-making, the beads task has rarely yielded elevated errorrates in delusional patients (Garety \& Freeman, 1999). As such, 'rushing' may aid in bridging the gap between the beads task findings and more real-to-life and ill-defined problems which are more likely to elicit elevated error rates.

\section{Limitations and Future Research}

Notably, these findings must be qualified owing to their preliminary nature, specifically due to use of a small analogue sample. Nevertheless, use of this sample renders medication and decompensation effects unlikely (Claridge, 1988), and suggests that these biases present in premorbid delusional ideation, thus potentially predating the disorder.

The 'rushing' measure has not been previously demonstrated in patient populations and derives from an unvalidated single-item response. Hence, the effect of rushing on beads task performance requires further investigation in a larger patient sample and using more robust multiple-item measures. Given the potential effect on Bayesian reasoning (see Heit, 1998), it may even be of interest to include it as a variable on other reasoning paradigms involving hypothesis-testing (see Dudley \& Over, 2003).

Despite ample data indicating independence of JTC and premorbid and current IQ or working memory functioning (e.g., Moritz \& Woodward, 2005), there are some findings to the contrary (e.g., Van Dael et al., 2006). Therefore, these measures could be included in a future multiple jar variant task.

\section{CONCLUSION}

To summarize, the present study provides consistent evidence for a tendency of delusion-prone 
individuals to strike decisions after collecting less data than controls, even when multiple hypotheses (i.e., containers) were available. As such, hasty decision-making emerged as somewhat independent of increased ambiguity, thus contradicting an LA account of JTC findings (c.f. Moritz et al., 2007). In addition, neither IU nor NFC seemed to represent substantial factors contributing to JTC. Merely rushing was able to explain some, but not most of the variance. As such, these findings provide support for the pertinence of minimal data gathering to delusions under various conditions, potentially owing to misperceived threat (Dudley \& Over, 2003) or avoidance of decision-making based on sequential information (Young \& Bentall, 1995). These effects were specific to delusion-prone populations, and were not explained by general psychopathology.

\section{REFERENCES}

Allen, P., Freeman, D., Johns, L., \& McGuire, P. (2006). Misattribution of self-generated speech in relation to hallucinatory proneness and delusional ideation in healthy volunteers. Schizophrenia Research, 84, 281-288.

Antony, M.M., Bieling, P.J., Cox, B.J., Enns, M.W., \& Swinson, R.P. (1998). Psychometric properties of the 42-item and 21-item versions of the Depression Anxiety Stress Scales in clinical groups and a community sample. Psychological Assessment, 10, 176-181.

Beck, A.T., \& Rector, N.A. (2005). Cognitive approaches to schizophrenia: Theory and therapy. Annual Review of Clinical Psychology, 1, 577-606.

Bentall, R., \& Swarbrick, R. (2003). The best laid schemas of paranoid patients: Autonomy, sociotrophy and need for closure. Psychology and Psychotherapy, 76, 163-171.

Boyd, T., \& Gumley, A. (2007). An experiential perspective on persecutory paranoia: A grounded theory construction. Psychology and Psychotherapy: Theory, Research E Practice, 80, 1-22.

Broome, M.R., Johns, L.C., Valli, I., Woolley, J.B., Tabraham, P., Brett, C., Valmaggia, L., Peters, E., Garety, P.A., \& McGuire, P.K. (2007). Delusion formation and reasoning biases in those at clinical high risk for psychosis. British Journal of Psychiatry, 191 (Suppl. 51), s38-s42.

Buchy, L., Woodward, T.S., \& Liotti, M. (2007). A cognitive bias against disconfirmatory evidence (BADE) is associated with schizotypy. Schizophrenia Research, 90, 334-337.

Buhr, K., \& Dugas, M.J. (2002). The intolerance of uncertainty scale: Psychometric properties of the English version. Behaviour Research and Therapy, 40, 931-945.

Claridge, G.S. (1988). Schizotypy and schizophrenia. In P. Bebbington \& P. McGuffin (Eds.), Schizophrenia: The major issues (pp.187-200). Oxford: Heinemann Professional.
Colbert, S.M., \& Peters, E.R. (2002). Need for closure and jumping-to-conclusions in delusion-prone individuals. Journal of Nervous and Mental Disease, 190, 27-31.

Colbert, S.M., Peters, E.R., \& Garety, P.A. (2006). Need for clusure and anxiety in delusions: A longitudinal investigation in early psychosis. Behaviour Research and Therapy, 44, 1385-1396.

Crawford, J.R., \& Henry, J.D. (2003). The Depression Anxiety Stress Scales (DASS): Normative data and latent structure in a large non-clinical sample. British Journal of Clinical Psychology, 42, 111-131.

Dudley, R.E.J., John, C.H., Young, A.W., \& Over, D.E. (1997a). Normal and abnormal reasoning in people with delusions. British Journal of Clinical Psychology, 36, 243-258.

Dudley, R.E.J., John, C.H., Young, A.W., \& Over, D.E. (1997b). The effect of self-referent material on the reasoning of people with delusions. British Journal of Clinical Psychology, 575-584.

Dudley, R.E.J., \& Over, D.E. (2003). People with delusions jump to conclusions: a theoretical account of research findings on the reasoning of people with delusions. Clinical Psychology \& Psychotherapy, 10, 263-274.

Fear, C.F., \& Healy, D. (1997). Probabilistic reasoning in obsessive-compulsive and delusional disorders. Psychological Medicine, 27, 199-208.

Fine, C., Gardner, M., Craigie, J., \& Gold, I. (2007). Hopping, skipping or jumping to conclusions? Clarifying the role of the JTC bias in delusions. Cognitive Neuropsychiatry, 12, 46-77.

Fischhoff, B., \& Beyth-Marom, R. (1983). Hypothesis evaluation from a Bayesian perspective. Psychological Review, 90, 239-260.

Fraser, J., Morrison, A.P., \& Wells, A. (2006). Cognitive processes, reasoning biases and persecutory delusions: A comparative study. Behavioural and Cognitive Psychotherapy, 34, 421-435.

Freeman, D., \& Garety, P.A. (2004). Paranoia: The psychology of persecutory delusions. Hove: Psychology Press.

Freeman, D., Garety, P.A., Kuipers, E., Colbert, E., Jolley, S., Fowler, D., Dunn, G., \& Bebbington, P. (2006). Delusions and decision-making style: Use of the Need for Closure Scale. Behaviour Research and Therapy, 44, 1147-1158.

Freeston, M.H., Rhéaume, J., Letarte, H., Dugas, M.J., \& Ladouceur, R. (1994). Why do people worry? Personality and Individual Differences, 17, 791-802.

Frost, R.O., \& Shows, D.L. (1993). The nature and measurement of compulsive indecisiveness. Behaviour Research and Therapy, 31, 683-692.

Garety, P.A., Bebbington, P., Fowler, D., Freeman, D., \& Kuipers, E. (2007). Implications for neurobiological research of cognitive models of psychosis: A theoretical paper. Psychological Medicine, 37, 1377-1391.

Garety, P.A., Freeman, D., Jolley, S.J., Dunn, G., Bebbington, P.E., Fowler, D.G., Kuipers, E., \& Dudley, R. (2005). Reasoning, emotions, and delusional conviction in psychosis. Journal of Abnormal Psychology, 114, 373-384.

Garety, P.A., \& Freeman, D. (1999). Cognitive approaches to delusions: A critical review of theories and evi- 
dence. British Journal of Clinical Psychology, 38, 113154.

Garety, P.A., \& Hemsley, D.R. (1994). Delusions: Investigations into the psychology of delusional reasoning. Hove: Psychology Press.

Garety, P.A., Hemsley, D.R., \& Wessely, S. (1991). Reasoning in deluded schizophrenic and paranoid patients: Biases in performance on a probabilistic inference task. Journal of Nervous and Mental Disease, 179, 194-201.

Green, M.J., Williams, L., \& Davidson, D. (2001). Processing of threat-related affect is delayed in delusionprone individuals. British Journal of Clinical Psychology, 40, 157-165.

Harvey, A., Watkins, E., Mansell, W., \& Shafran, R. (2004). Cognitive behavioural processes across psychological disorders: A transdiagnostic approach to research and treatment. New York: Oxford University Press.

Heit, E. (1998). Influences of prior knowledge on selective weighting of category members. Journal of Experimental Psychology: Learning, Memory, and Cognition, 24, 712-731.

Hemsley, D.R. (1987). An experimental psychological model for schizophrenia. In H. Hafner, W.F. Gattaz, \& W. Janzarik, (Eds.), Search for the causes of schizophrenia (Vol. 1, pp. 179-188). New York: Springer.

Hemsley, D.R. (2005). The development of a cognitive model of schizophrenia: Placing it in context. Neuroscience and Biobehavioral Reviews, 29, 977-988.

Hirschfeld, R.M.A., Williams, J.B.W., Spitzer, R.L., Calabrese, J.R., Flynn, L., Keck Jr., P.E., Lewis, L., McElroy, S.L., Post, R.M., Rapport, D.J., Russell, J.M., Sachs, G.S., \& Zajecka, J. (2000). Development and validation of a screening instrument for bipolar spectrum disorder: The Mood Disorder Questionnaire. American Journal of Psychiatry, 157, 1873-1875.

Holaway, R.M., Heimberg R.G., \& Coles, M.E. (2006). A comparison of intolerance of uncertainty in analogue obsessive-compulsive disorder and generalized anxiety disorder. Anxiety Disorders, 20, 158-174.

Huq, S.F., Garety, P.A., \& Hemsley, D.R. (1988). Probabilistic judgements in deluded and nondeluded subjects. Quarterly Journal of Experimental Psychology, 40, 801-812.

Kapur, S. (2003). Psychosis as a state of aberrant salience: A framework linking biology, phenomenology, and pharmacology in schizophrenia. American Journal of Psychiatry, 160, 13-23.

Kruglanski, A.W., \& Webster, D.M. (1996). Motivated closing of the mind: 'Seizing' and 'Freezing'. Psychological Review, 103, 263-283.

Kruglanski, A.W., Webster, D.M., \& Klem, A. (1993). Motivated resistance and openness to persuasion in the presence or absence of prior information. Journal of Personality and Social Psychology, 65, 861-876.

Laroi, F., \& van der Linden, M. (2005). Metacognitions in proneness towards hallucinations and delusions. Behaviour Research and Therapy, 43, 1425-1441.

Linney, Y.M., Peters, E.R., \& Ayton, P. (1998). Reasoning biases in delusion-prone individuals. British Journal of Clinical Psychology, 37, 285-302.

Lovibond, P.F., \& Lovibond, S.H. (1995). The structure of negative emotional states: Comparison of the Depres- sion Anxiety Stress Scales with the Beck Depression and Anxiety Inventories. Behaviour Research Therapy, 33, 335-343.

McKay, R., Langdon, R., \& Coltheart, M. (2006). Need for closure, jumping to conclusions, and decisiveness in delusion-prone individuals. Journal of Nervous and Mental Disease, 194, 422-426.

Marken, R.S. (2003). Error in skilled performance: A control model of prescribing. Ergonomics, 46, 1200-1214.

Menon, M., Pomarol-Clôtet, E., McKenna, P.J., \& McCarthy, R.A. (2006). Probabilistic reasoning in schizophrenia: A comparison of the performance of deluded and nondeluded schizophrenic patients and exploration of possible cognitive underpinnings. Cognitive Neuropsychiatry, 11, 521-536.

Moritz, S., \& Lincoln, T.M. (2007). Kognitive Korrelate des schizophrenen Wahns. Podcast retrieved from http:// www.uke.ani-hamburg.de/kliniken/psychiatrie/ downioads/klinik-psychiatvie-psychothevapie / Movitz_-_Wahn_Kognition_sewinar.pdf.

Moritz, S., \& Woodward, T.S. (2004). Plausibility judgment in schizophrenic patients: Evidence for a liberal acceptance bias. German Journal of Psychiatry, 7, 6674.

Moritz, S., \& Woodward, T.S. (2005). Jumping to conclusions in delusional and non-delusional schizophrenic patients. British Journal of Clinical Psychology, 44, 193-207.

Moritz, S., \& Woodward, T.S. (2006). A generalised bias against disconfirmatory evidence in schizophrenia. Psychiatry Research, 142, 157-165.

Moritz, S., Woodward, T.S., \& Hausmann, D. (2006). Incautious reasoning as a pathogenetic factor for the development of psychotic symptoms in schizophrenia. Schizophrenia Bulletin, 32, 327-331.

Moritz, S., Woodward, T.S., \& Lambert, M. (2007). Under what circumstances do people with schizophrenia jump to conclusions: A liberal acceptance account. British Journal of Clinical Psychology, 46, 127-137.

Mullen, R. (2003). Delusions: The continuum versus category approach. Australian and New Zealand Journal of Psychiatry, 37, 505-511.

Peters, E., \& Garety, P.A. (1996). The Peters et al. Delusions Inventory (PDI): New norms for the 21-item version. Schizophrenia Research, 18, 118-119.

Peters, E.R., \& Garety, P.A. (2006). Cognitive functioning in delusions: A longitudinal analysis. Behavioural Research and Therapy, 44, 481-514.

Peters, E.R., Joseph, S.A., Day, S., \& Garety, P.A. (2004). Measuring delusional ideation: The 21-item Peters et al. Delusions Inventory (PDI). Schizophrenia Bulletin, 30, 1005-1016.

Peters, E.R., Joseph, S.A., \& Garety, P.A. (1999). Measurement of delusional ideation in the normal population: Introducing the PDI (Peters et al. Delusions Inventory). Schizophrenia Bulletin, 25, 553-576.

Phillips, L.D., \& Edwards, W. (1966). Conservatism in a simple probabilistic inference task. Journal of Experimental Psychology, 72, 346-354.

Preti, A., Rocchi, M.B.L., Sisti, D., Mura, T., Manca, S., Siddi, S., Petretto, D.R., \& Masala, C. (2007). The 
psychometric properties of the Peters et al. Delusions Inventory: A receiver operating characteristic curve analysis. Comprehensive Psychiatry, 48, 62-69.

Van Dael, F., Versmissen, D., Jannsen, I., Myin-Germeys, I., van Os, J., \& Krabbendam, L. (2006). Data gathering: Biased in psychosis? Schizophrenia Bulletin, 32,341-351.

Warman, D.M., Lysaker, P.H., Martin, J.M., Davis, L., \& Haudenschield, S.L. (2007). Jumping to conclusions and the continuum of delusional beliefs. Behaviour Research and Therapy, 45, 1255-1269.

Warman, D.M., \& Martin, J.M. (2006). Jumping to conclusions and delusion-proneness: The impact of emotionally salient stimuli. The Journal of Nervous and Mental Disease, 194, 760-765.

Woodward, T.S., Moritz, S., Cuttler, C., \& Whitman, J.C. (2006). The contribution of a Cognitive Bias Against
Disconfirmatory Evidence (BADE) to delusions in schizophrenia. Journal of Clinical and Experimental Neuropsychology, 28, 605-617.

Young, H.F., \& Bentall, R.P. (1995). Hypothesis testing in patients with persecutory delusions: Comparison with depressed and normal subjects. British Journal of Clinical Psychology, 34, 353-369.

Young, H.F., \& Bentall, R.P. (1997). Probabilistic reasoning in deluded, depressed and normal subjects: Effects of task difficulty and meaningful versus nonmeaningful material. Psychological Medicine, 27, 455465.

Ziegler, M., Rief, W., Werner, S.-M., Mehl, S., \& Lincoln, T.M. (2008). Hasty decision-making in a variety of tasks: Does it contribute to the development of delusions? Psychology and Psychotherapy: Theory, Research and Practice, 81, 237-245. 